\title{
The benefits of manual therapy and active cervical exercises in pacients with cerviogenic headache
}

\author{
Manuela Lalu1*, Petru Mihancea1 ${ }^{1}$, Olivia Andreea Marcu² \\ 1. Doctoral School, University of Oradea, Romania \\ 2. Preclinics department, Faculty of Medicine and Pharmacy, University of Oradea, Romania
}

Introduction: Cervicogenic headache stems from a dysfunctional mechanism that frequently affects the upper region of the cervical spine, often involving multiple tissues connected with the occipital, submandibular, and orofacial region. Objectives: The main objective of this study is to demonstrate the therapeutic effectiveness of a personalized program of manual therapy and specific exercises in patients with cervicogenic headaches. Material and Methods: The study included 44 patients. Specific functional tests of the cranio-cervical-mandibular complex, namely the evaluation of the cranio-cervical-mandibular complex (according to Rocabado), cervical flexion-rotation test, cervical flexor endurance test, cervical extensors endurance test, and palpation of trigger points and cervical spine dynamics, were employed. Results: The analysis of demographic data shows a significantly increased prevalence of headache among women. The symptomatology that almost always accompanies episodes of cervicogenic headache is localized neck pain. The intensity and frequency of the symptoms are strongly associated with alterations in the dynamics of the upper cervical spine and chronic sleep disorders. Conclusion: Cranio-cervical manual therapy has proven effective in patients with cervicogenic headaches. The implementation of a strategy based on manual therapy and active exercise had a superior statistical and clinical result compared to the exclusively manual therapeutic approach.

Keywords: cervicogenic headache, manual therapy, cranio-cervical-mandibular, headache, active exercise

Received 15 June 2021 / Accepted 21 July 2021

\section{Introduction}

Across the world, headaches are recognized more and more as one of the many reasons for disability, with an increasingly pervasive presence amongst the general population. It is estimated that around $50 \%$ of people worldwide experience episodes of headaches of varying duration and intensity [1] at least once a year. On a global scale, headaches rank amongst the first three causes of disability [2].

The International Headache Society classifies headaches into three major categories:

- Primary headaches, which do not depend on any specific pathology and whose etiology remains unknown, including migraines, tension headaches, and cluster headaches.

- Secondary headaches, which are a consequence of other pathologies, including cervicogenic headaches and temporomandibular headaches, which are amongst the most common forms found in our clinical practice.

Painful cranial neuropathies and other types of orofacial pain [3].

The most common type of headache is a tension-type headache with a prevalence of $38 \%$, followed by migraine with a prevalence of $10 \%$, and cervicogenic headache of $2.5-4.1 \%[4]$.

The cervicogenic headache is a form of secondary headache caused by cervical dysfunction especially of the first three cervical vertebrae. It should be noted that the lower segments can contribute to its genesis [25]. Diagnosis cri-

* Correspondence to: Manuela Lalu

E-mail: manu_mldv@yahoo.com teria for cervicogenic headache include headaches associated with cervical pain and stiffness of the cervical spine. These consist of unilateral pain starting from the posterior cervical region, with varying duration and intensity, and with possible atypical irradiation in the anterior lateral area of the skull and/or the face [3-5].

The convergence theory supports the reason for the perception of this pain as headache [6]. Neurophysiology and neuroanatomy research has shown the existence of convergences between nociceptive afferents of the cervical spinal nerves and those in the trigeminal territory occurring in the trigeminocervical nucleus located in the upper segment of the cervical spine [6,7]. Also relevant in this description is the convergence of the sensory-motor fibers of the accessory nerve (XI) [8] with the roots of the superior cervical nerves and the trigeminal nerve. These connections account for the pain in the upper trapezius muscle, sternocleidomastoid muscles, face, and neck.

Given that there is also a correlation between the cervicogenic headache and cervical muscular imbalance at the level of deep flexors and extensors of the neck $[4,9,10]$, a clear and systematic approach to this type of headache is required both from a diagnostic, therapeutic and management point of view subsequent to these patients.

Although the cervicogenic headache has a low prevalence compared to other types of headaches, it has a major impact on the quality of life [11]. Most commonly, these patients experience episodes of headaches, but the difficulty in identifying the causes involved in the onset of this symptom leads to patients becoming chronic ones. The scientific community states that a possible explanation could be the sensitization of the central nervous system 
(CNS) due to the prolonged stimulation of nociceptors in the pericranial myofascial tissues [12-16]. Specific manual therapy (MT) is meant to act on these structures in order to reduce mechanical stress $[17,18]$.

Comorbidities of people suffering from headaches include anxiety, depression, and insomnia, thus making the socio-economic impact even greater [19-23].

The diagnostic and therapeutic approach must take into account all biomechanical and psychological aspects in order to make a correct differential diagnosis [24,25].

The tendency of the scientific community is to find quick, affordable, and easy-to-adopt solutions that keep under control, or even alleviate, these syndromes given that there are some known underlying pathophysiological mechanisms.

\section{Objectives}

The objective of our study was to demonstrate that a mixed therapeutic approach (MT specific to the cranio-cervicalmandibular (CCM) area combined with a program of active exercises specific to this region), adapted to the patient's needs has an increased efficiency compared to the single approach (MT). This is possible through a causeoriented intervention that causes sensitization of the CNS with consequences that affect the patients' well-being.

The hypothesis of the study is that only awareness of the risk factor and active involvement with constant monitoring ensures comfort and an improvement in the quality of life of patients with cervicogenic headache. This hypothesis is based on results from the clinical practice of the main author in the past 5 years. The premise of the study was that the mixed approach is the key to significant improvement and alleviation until the symptomatology of these patients is resolved.

\section{Material and Methods}

The study began by monitoring a group of patients with headaches who contracted the services of the physiotherapy department within the Linea Medica Clinic in Oradea, Romania, during May-December 2019.

The criteria for inclusion in the study were:

- Cervicogenic headaches based on clinical and functional differential diagnosis

- Patients initially evaluated by a specialist doctor (neurologist, physical medicine and rehabilitation)

- Patients with chronic pain lasting more than 3 months

- Consent to be included in the study

- Commitment to follow the entire program (3 months duration, 2 weekly sessions), plus a follow-up session ( 3 months and 7 months after the end of the program)

The exclusion criteria were:

- Patients with acute headaches

- Patients with psychiatric pathologies

- Patients with cervical fractures undergoing consoli- dation

- Pathologies that require constant drug treatment.

A group of 49 patients (10 men and 39 women) between the ages of 16 and 63 were initially selected. All patients have expressed written consent to participate in this study and showed compliance with the requirements of the study. We must add that for all minor patients we -obtained the written consent of their legal representatives regarding their participation in our study and the use of the data for scientific purposes. A few patients, four men and one woman, chose other solutions for treatment, which led to their compliance being misaligned with our study. The remaining group of 44 patients (6 men and 38 women) was treated and monitored according to the study criteria.

All subjects were assessed clinically and biomechanically. The clinical evaluation consisted of anamnesis, objective examination by regions, and interdisciplinary evaluation (neurology, physical medicine and rehabilitation, neurosurgery, ophthalmology). The biomechanical evaluation included specific tests: evaluation of the cranio-cervicalmandibular complex (CCM) (Rocabado) [26] including cervical rotation-flexion test (FRT) [27], cervical flexor strength test (FST), cervical extensor strength test (EST) $[28,29]$. Pain severity was assessed using visual analog scale (VAS). The participants were asked to rate pain from 1 to 10 , with a minimum of 1 and a maximum of 10 . The Pittsburgh Sleep Quality Index [30] was used to assess sleep duration and quality.

The importance of manual therapy and active exercises was explained to all patients. During treatment, some patients assumed non-compliance with the physical activity program, yet maintaining compliance with MT. Thus, the idea of a comparative study between the two groups emerged:

- Group 1 (G1) consisting of 24 patients had specific MT according to internationally validated concepts and recognized by the International Society for Headaches. In addition, they went through a program of targeted active exercises for the cervical region. This program was customized according to the patients' needs following the initial evaluation.

- Group 2 (G2) consisting of 20 patients had only specific MT.

All 44 patients benefited from two sessions of specific MT per week, and those from group 1 performed, two additional exercise sessions of 15 minutes per day at home (one session during the day at any one given time; the other at night before bedtime). It is worth mentioning that all patients voluntarily chose not to benefit from drug therapy or other type of physiotherapy in agreement with the attending specialist doctor.

Patients were monitored both objectively and subjectively:

- Objectively, by periodically re-applying the functional tests used in the initial evaluation

- Subjectively, by self-monitoring i.e quantifying the 
pain on a scale of 1 to 10 according to a visual analogue scale (VAS), plus the subjective description of efficiency in general (desire and ease of involvement in daily intellectual and physical activities, changes in sleep quality). It should also be noted that all subjects complained of sleep disorders, more precisely changes in terms of quality and quantity of sleep (information obtained in the initial history using Pittsburgh Sleep Quality Index (PSQI) [30]. The evaluation based on this scale showed that a large number of patients experienced insomnia, since they had issues in terms of falling asleep. Sleep duration was not the desired one and, due to poor sleep quality, they were experiencing low energy levels all day.

Both approaches were supplemented with the patients' education (clarification of the mechanisms of headache production, risk factors, counseling in order to apply ergonomic measures to reduce the symptomatology and maintain the results of the therapy).

Statistical analysis was performed using the statistical package SPSS24. The descriptive characteristics of the quantitative data were reported as mean value \pm SD (standard deviation), while the qualitative ones are expressed as percentages. Parameter differences between the two groups of patients were assessed based on independent t-tests for quantitative data and Chi-Square tests for the qualitative, while the differences among "stages" of variables were assessed using ANOVA. The significance level was set at $\mathrm{p}<0.05$, otherwise mentioned.

\section{Results}

The analysis of the demographic data shows the overwhelming prevalence of women in both groups $(95.8 \%$ in G1, 75\% in G2), with a significant difference in the distribution of the population. There is a significant difference in the mean ages of the two groups, $30.5+11.15$ versus $38.1+6.35$. Patient location is mainly urban in both groups, 23 (95.8\%) on the first group and 17 (85\%) in the second one, with no significant differences.

Table II outlines the changes in all variables between the two groups of patients and in between three visits to the
Table I. Demographic data

\begin{tabular}{|c|c|c|c|c|c|}
\hline \multirow{2}{*}{$\begin{array}{l}\text { Vari- } \\
\text { able }\end{array}$} & \multicolumn{2}{|c|}{ Group 1} & \multicolumn{2}{|l|}{ Group 2} & \multirow{2}{*}{$p$-value } \\
\hline & mean+stdev & 24 (100\%) & mean+stdev & 20 (100\%) & \\
\hline Age & $30.5+11.15$ & & $38.1+6.35$ & & 0.007 \\
\hline Sex & & & & & 0.04 \\
\hline - male & & $1(4.2 \%)$ & & $5(25 \%)$ & \\
\hline - female & & 23 (95.8\%) & & 15 (75\%) & \\
\hline Area & & & & & 0.21 \\
\hline - rural & & $1(4.2 \%)$ & & $3(15 \%)$ & \\
\hline - urban & & 23 (95.80\%) & & 17 (85\%) & \\
\hline
\end{tabular}

doctor, for each group. VAS results show a decrease from G1 to G2 at all three visits, but statistically significant only for the first visit at a 0.1 significance level, with an average decrease of 0.81. Pain frequency decreased at the first two visits, with a significant difference only at the first visit of 0.88 points and 0.07 at the second one, followed by an increase of 0.33 points at the last visit. There are no major differences in FRT, FST and EST at the first visit and slight increases in the next two visits. When it comes to insomnia, the level of intensity is decreasing from G1 to G2 in the first two visits, significantly different at the second visit when a 0.1 level of significance is considered.

Comparisons among the intensity levels of the studied aspects in the three visits indicate significant differences in VAS levels, pain frequency, FRT, FST, EST in both groups, while insomnia is significantly different only in G1 patients.

Table III outlines all differences among each two visits, according to Scheffe's test. For the G1 patients, the VAS variable presents significant differences between visits 1 and 3, and then 2 and 3. Pain frequency, FRT, FST and EST have significant differences among each two visits, while in insomnia, the differences are significant only between visit 1 to 3 , and 2 to 3 . For G2, there are significant differences among all two visits for VAS variable and pain frequency. In FRT there is a significant difference between visit 1 to 3 at a 0.1 level of significance and at 0.05 level between visit 2 to 3 . FST and EST are significantly different

Table II. Variable comparisons

\begin{tabular}{|c|c|c|c|c|c|c|c|c|}
\hline \multirow{2}{*}{ Variable } & \multicolumn{3}{|c|}{ VAS } & \multicolumn{5}{|c|}{ Pain frequency } \\
\hline & 1 & 2 & 3 & $p$-value & 1 & 2 & 3 & p-value \\
\hline G1 & $5.66 \pm 1.46$ & $2.12 \pm 1.51$ & $1.66 \pm 0.11$ & 0 & $4.33 \pm 0.96$ & $1.37 \pm 1.09$ & $0.12 \pm 0.44$ & 0 \\
\hline G2 & $4.85 \pm 1.26$ & $1.9 \pm 1.65$ & $0.88 \pm 0.19$ & 0 & $3.45 \pm 0.51$ & $1.3 \pm 1.08$ & $0.45 \pm 0.82$ & 0 \\
\hline \multirow[t]{3}{*}{$\mathrm{p}$-value } & 0.057 & 0.64 & 0.13 & & 0 & 0.82 & 0.126 & \\
\hline & \multicolumn{3}{|c|}{ FRT } & \multicolumn{5}{|c|}{ FST } \\
\hline & 1 & 2 & 3 & $\mathrm{p}$-value & 1 & 2 & 3 & $\mathrm{p}$-value \\
\hline G1 & $1 \pm 0$ & $0.7 \pm 0.46$ & $0.08 \pm 0.28$ & 0 & $1 \pm 0$ & $0.70 \pm 0.46$ & $0.08 \pm 0.28$ & 0 \\
\hline G2 & $1 \pm 0$ & $0.75 \pm 0.44$ & $0.25 \pm 0.44$ & 0 & $1 \pm 0$ & $0.75 \pm 0.44$ & $0.25 \pm 0.44$ & 0 \\
\hline \multirow[t]{3}{*}{$\mathrm{p}$-value } & 1 & 0.76 & 0.157 & & 1 & 0.76 & 0.157 & \\
\hline & \multicolumn{3}{|c|}{ EST } & \multicolumn{5}{|c|}{ Insomnia } \\
\hline & 1 & 2 & 3 & p-value & 1 & 2 & 3 & p-value \\
\hline G1 & 1 & $0.70 \pm 0.46$ & $0.08 \pm 0.28$ & 0 & $0.6 \pm 0.48$ & $0.58 \pm 0.50$ & $0.20 \pm 0.41$ & 0.003 \\
\hline G2 & 1 & $0.75 \pm 0.44$ & $0.25 \pm 0.44$ & 0 & $0.3 \pm 0.47$ & $0.3 \pm 0.47$ & $0.20 \pm 0.41$ & 0.722 \\
\hline$p$-value & 1 & 0.76 & 0.157 & & 1 & 0.062 & 0.947 & \\
\hline
\end{tabular}

G1 - group 1; G2 - group 2 
between visits 1 to 3 and 2 to 3 . There are no significant differences in insomnia.

\section{Discussion}

In most cases, the patient reaches us with a chronic headache often due to a lack of proper education related to the use of manual therapy. This tends to happen despite the fact that the specialized literature states this is the most common type of physical therapy used for headaches in most countries [31].

The approach presented in our study and used in our daily practice is based on patient education, manual therapy specific to the CCM region and a set of physical exercises specific to this area (exercises that also include a routine stretching of the suboccipital muscles) with direct impact on the tissues involved in the pericranial nociceptive mechanism.

Our study suggests that the cervicogenic headache occurs more frequently in women than men, which matches the data from the specialized literature [25]. There is also a low median age (lower than the average of international studies [32]).

Significant reduction of pain (in intensity and frequency) is due to decreased stress in the suboccipital and pericranial tissues by inhibiting nociceptors. Maintaining these results is a consequence of physical exercises. The results obtained through our study suggest that manual therapy combined with physical exercises are more effective in reducing the pain and improving the cervical spine mobility compared to a singular approach. These findings are also

Table III. Mean differences among visits

\begin{tabular}{|c|c|c|c|c|c|c|}
\hline \multirow{3}{*}{$\begin{array}{l}\text { Variable } \\
\text { VAS }\end{array}$} & \multirow{2}{*}{\multicolumn{2}{|c|}{ Stage }} & \multicolumn{2}{|c|}{ Group 1} & \multicolumn{2}{|c|}{ Group 2} \\
\hline & & & \multirow{2}{*}{$\begin{array}{c}\begin{array}{c}\text { Mean } \\
\text { diff }\end{array} \\
0\end{array}$} & \multirow{2}{*}{$\frac{\text { p-value }}{1}$} & \multirow{2}{*}{$\begin{array}{c}\text { Mean } \\
\text { Diff }\end{array}$} & \multirow{2}{*}{$\frac{\text { p-value }}{0}$} \\
\hline & 1 & 2 & & & & \\
\hline & & 3 & 1.95 & 0 & 4.35 & 0 \\
\hline & 2 & 3 & 1.95 & 0 & 1.4 & 0.005 \\
\hline \multirow[t]{3}{*}{ Pain Frequency } & 1 & 2 & 2.95 & 0 & 2.15 & 0 \\
\hline & & 3 & 4.2 & 0 & 3 & 0 \\
\hline & 2 & 3 & 1.25 & 0 & 0.85 & 0 \\
\hline \multirow[t]{3}{*}{ FRT } & 1 & 2 & 0.29 & 0.008 & -0.3 & 0.48 \\
\hline & & 3 & 0.91 & 0 & 0.55 & 0.095 \\
\hline & 2 & 3 & 0.62 & 0 & 0.85 & 0.005 \\
\hline \multirow[t]{3}{*}{ FST } & 1 & 2 & 0.29 & 0.008 & 0.25 & 0.102 \\
\hline & & 3 & 0.91 & 0 & 0.75 & 0 \\
\hline & 2 & 3 & 0.62 & 0 & 0.5 & 0 \\
\hline \multirow[t]{3}{*}{ EST } & 1 & 2 & 0.29 & 0.008 & 0.25 & 0.102 \\
\hline & & 3 & 0.91 & 0 & 0.75 & 0 \\
\hline & 2 & 3 & 0.62 & 0 & 0.5 & 0 \\
\hline \multirow[t]{3}{*}{ Insomnia } & 1 & 2 & 0.08 & 0.82 & 0 & 1 \\
\hline & & 3 & 0.45 & 0.005 & 1 & 0.78 \\
\hline & 2 & 3 & 0.37 & 0.026 & 1 & 0.78 \\
\hline
\end{tabular}

FRT - rotation-flexion test; FST - cervical flexor strength test; EST - cervical extensor strength test endorsed by the specialized literature [33]. Equally important, these exercises ensure the re-education of the deep cervical muscles.

The quality of sleep increases due to pain alleviation, and thus an improvement of the general physical and mental state.

Considering the impact and the results in the short, mid and long term, the minimal costs, and the ease with which a targeted program can be customized and implemented even in a small group we suggest extending the study to a larger group. The results of this study show that as the painful syndrome decreases, the patient's compliance increases since now they trust the non-invasive measures proposed by the medical specialists. For this reason, we suggest re-evaluating the therapeutic strategies of these patients in the long and even longer term in close collaboration with the specialist doctor.

The limits of the study are due to the small number of subjects partaking in this research, on account of a combination of factors:

- the prevalence of cervicogenic headache in all cases of headache is low

- the patients' skepticism towards an "apparently" too simple approach - here we are referring to patients with chronic pain extended over a number of years, and patients who received countless therapeutic treatments with unsatisfactory results

- the difficulty of active involvement in one's own therapeutic program - the effort to maintain a rigorous program (constant presence in manual therapy and performing exercises at home).

That said we consider that the practical value of the obtained results is encouraging.

\section{Conclusions}

- Specific manual therapy, along with a personalized exercise program based on the patients' needs determines the significant short and mid-term alleviation of chronic cervical pain and headache, and a longterm impact on the recurrence of pain.

- To achieve positive outcomes, it is extremely important to detect the predisposing and triggering factors, which is only possible by creating a trusting relationship (therapeutic alliance) with the patient.

- The reorganization of the therapeutic strategy, which includes the patient's education, leads to a clearer understanding of the phenomena. This, in return, results in the maintenance of the mechanisms of the headache and in the long term, offers an important psychological comfort.

- Chronic headache has important personal, social, and economic consequences for the patient, with changes in the adaptation of the chronic pain for the entire lifestyle.

- These types of therapy have a low cost and can be implemented quickly, right after their clinical diagno- 
sis. In the long run, they can reduce the associated disability, increase the workplace performance, and decrease healthcare expenses.

\section{List of abbreviations}

CNS -central nervous system

MT - manual therapy

CCM - cranio-cervical-mandibular

$\mathrm{G} 1$ - group 1

G2 - group 2

FRT- rotation-flexion test

FST - cervical flexor strength test

EST -cervical extensor strength test

\section{Author contributions}

ML: (Conceptualization; Methodology; Investigation; Data collection; Data interpretation; Conception and design of the manuscript)

PM: (Revising of the manuscript, final approval of the version to be published)

OAM: (Data analysis and interpretation)

\section{Conflict of interest}

None to declare.

\section{References}

1. Stovner L, Hagen K, Jensen R, et al. The global burden of headache: a documentation of headache prevalence and disability worldwide. Cephalalgia 2007;27:193-210.

2. Timothy J, Steiner, Gretchen L, et al. Headache disorders are third cause of disability worldwide. J Headache Pain 2015;16:58

3. International Headache Society. The International Classification of Headache Disorders, 3rd edition. Cephalalgia 2018;38:1-211

4. Toby H, Kathy B, Diana H. Clinical Evaluation of Cervicogenic Headache: A Clinical Perspective. J Man Manip Ther 2008;16: 73-80.

5. Sjaastad O, Fredriksen T, Pfaffenrath V. Cervicogenic Headache: Diagnostic Criteria. Headache 1990;30 :725-6

6. Nicholas $\mathrm{H}$, Hans $\mathrm{V}$, Oliver $\mathrm{H}$, Kris C. Understanding Cervicogenic Headache. Anesth Pain 2012:2:3-4

7. BogdukN. Cervicogenic headache: anatomic basis and pathophysiologic mechanisms. Curr Pain Headache Rep 2001;5:382-6.

8. David M. Cervicogenic Headache : A Review of Diagnostic and Treatment Strategies. Am Osteopath Assoc 2005;105:16S-22S

9. Inae C, Susan A, David J. Cervical musculoskeletal impairments in cervicogenic headache: a systematic review and a meta-analysis. Physical Therapy Reviews 2008;13:149-166

10. Xiao-Ying $\mathrm{Y}$, Sheng-Bo $\mathrm{Y}$, Cong $\mathrm{L}$, et al. Correlation Between Chronic Headaches and the Rectus Capitis Posterior Minor Muscle: A Comparative Analysis of Cross-Sectional Trail. Cephalalgia 2017;37:1051-1056

11. Hans A, Inge L, Suzanne G, Alfons G, Wilhelm E. Quality of life of patients with cervicogenic headache: a comparison with control subjects and patients with migraine or tension-type headache. Headache 2003;43:1034-41
12. Kjersti A, Ragnhild B, Christofer L, Michael B. Pericranial tenderness in chronic tension-type headache: the Akershus population-based study of chronic headache. J Headache Pain 2014;15:58

13. Lars B, David B, Sait A. Mechanisms of Tension-Type Headache and Their Relevance to Management. Handbook of Headache 2011;pp 283-294.

14. Cezar F, Cuadrado M, Arendt-Nielsen L, Ge H, Pareja J. Increased pericranial tenderness, decreased pressure pain threshold, and headache clinical parameters in chronic tension-type headache patients. Clin J Pain 2007;23:346-52.

15. Aaseth K, Grande R, Lundqvist C, Russell M. Pericranial tenderness in chronic tension-type headache: the Akershus population-based study of chronic headache. J Headache Pain 2014:5:15:58.

16. Cezar F. Myofascial Head Pain. Curr Pain Headache Rep. 2015;19:28.

17. Aleksander $\mathrm{C}$, Michael B. Manual therapies for cervicogenic headache: a systematic review. J Headache Pain 2012;13:351-359

18. Craig S, David W, Jon A. A critical review of manual therapy use for headache disorders: prevalence, profiles, motivations, communication and self-reported effectiveness. BMC Neurology 2017;17:61

19. Shuu-Jiun W, Ping-Kun C, Jong-Ling F. Comorbidities of Migraine. Front Neurol 2010;1:16.

20. Luigi F, Andrea G, Romina C. Sleep disorder-related headaches. Neurol Sci 2019;40:107-113

21. Nu C, Jason D, Lee P. The epidemiology and comorbidities of migraine and tension-type headache. Techniques in Regional Anesthesia and Pain Management 2012; Volume 16, Issue 1:Pages 4-13

22. César F, Juan J, Maria P, Paula P, Margarita C. Sleep disturbances in tension-type headache and migraine. Ther Adv Neurol Disord 2017;6;11:1756285617745444

23. Andrijauskis D, Ciauskaite J, Vaitkus A, Pajediene E. Primary Headaches and Sleep Disturbances: A Cause or a Consequence?. J Oral Facial Pain Headache 2019:34:61-66

24. Sarah H, Darren H, Alison M. Use of orthopedic manual physical therapy to manage chronic orofacial pain and tension-type headache in an adolescent. J Man Manip Ther 2014;22:51-58.

25. Phil P. CERVICOGENIC HEADACHES: AN EVIDENCE-LED APPROACH TO CLINICAL MANAGEMENT. Int J Sports Phys Ther 2011;6:254-266.

26. Mariano R. The Importance of Soft Tissue Mechanics in Stability and Instability of the Cervical Spine: A Functional Diagnosis for Treatment Planning. Cranio 1987;5:130-138

27. Ogince M, Toby H, Robinson K, Blackmore AM. The diagnostic validity of the cervical flexion-rotation test in $\mathrm{C} 1 / 2$-related cervicogenic headache. Man Ther. 2007;12:256-62.

28. Jull G, O'Leary SP, Falla D. Clinical Assessment of the Deep Cervical Flexor Muscles: The Craniocervical Flexion Test. J. Manip. Physiol. Ther 2008;31:525-533

29. Luedtke K, Allers A, Schulte LH, May A. Efficacy of interventions used by physiotherapists for patients with headache and migraine-systematic review and meta-analysis. Cephalalgia 2016;36:474-92.

30. Mollayeva T, Pravheen T, Burton K, Mollayeva S, Shapiro C, Calantonio A. The Pittsburgh sleep quality index as a screening tool for sleep dysfunction in clinical and non-clinical samples: A systematic review and meta-analysis. Sleep Med Rev. 2016 Feb;25:52-73

31. Moore CS, Sibbritt DW, Adams J. A critical review of manual therapy use for headache disorders: prevalence, profiles, motivations, communication and self-reported effectiveness. BMC Neurol. 2017 Mar 24;17(1):61

32. Sjaastad $\mathrm{O}$, Bakketeig $\mathrm{L}$. Prevalence of Cervicogenic Headache: Vågå Study of Headache Epidemiology. Acta Neurol Scand. 2008;117:17380

33. Cumplido-Trasmonte C, Fernández-GonzálezF, Alguacil-Diego I, Molina-Rueda F. Manual therapy in adults with tension-type headache: a systematic review. Neurología (English Edition). 2020 